\title{
Silver Nanoparticles Effect on Antimicrobial and Antifungal Activity of New Heterocycles
}

\author{
Nadia G. Kandile, ${ }^{*}$ Howida T. Zaky, Mansoura I. Mohamed, and Hemat M. Mohamed \\ Chemistry Department, Faculty of Women, Ain Shams University, Heliopolis, 11757 Cairo, Egypt \\ ${ }^{*}$ E-mail:nadiaghk@yahoo.com \\ Received April 12, 2010, Accepted September 16, 2010
}

\begin{abstract}
In this study 1-[4-(2-methoxy benzyl)-6-aryl-pyridazin-3(2H)-ylidene] hydrazines were used for the synthesis of new heterocyclic systems such as thiazolidine, phthalazine, pyrazolo, tetrazolo, hydrazide and new pyridazine derivatives to explore the effect of silver nanoparticles on their biological activity efficiency. Structures of the new heterocycles were characterized by the aid of several analytical techniques including; ${ }^{1} \mathrm{H}-\mathrm{NMR}$, FTIR and mass spectra. Silver nanoparticles were synthesized by a simple methodology and the formation of silver nanoparticles was confirmed by transmission electron microscopy (TEM) and UV studies. Most of the new prepared heterocycles were evaluated in vitro as new antimicrobial agents. Combination effects of the silver nanoparticles on the antimicrobial activity of the new heterocycles were investigated using the disk diffusion method. Compound 10a exhibited the strongest enhancing effect of silver nanoparticles solution against Aspergillus flavus and Candida albicans.
\end{abstract}

Key Words: Silver nanoparticles, Pyridazine, Antimicrobial, Antifungal and (TEM)

\section{Introduction}

Nanoparticles are emerging materials that have a broad range of applications and notable characteristics different from those of bulk materials. They often have specific optical and electronic properties ${ }^{1}$ and chemical reactivity. ${ }^{2}$ Silver nanoparticles (Ag-NPs or nanosilver) are one of the most widely used nanoparticles, most notably serving as an antimicrobial agent for medical applications. The toxicity of nanosilver may be explained by the interaction of nanoparticles with microbes involving silver ion release and particle cellular internalization. ${ }^{3,4}$ Sizedependent toxicity of nanosilver ${ }^{5,6,7}$ supports the mode of action of Ag-NPs. The nanosilver toxicity is species specific. Smallsized Ag-NPs can inhibit nitrifying bacterial growth more than silver ions at the same total silver concentrations. ${ }^{6,8}$ However, Ag-NPs are not as effective as Ag ions in killing Escherichia coli. ${ }^{8}$ Over the past decade; there has been a strong push towards the development of silver-containing materials for commercial use that exhibit antimicrobial or bactericidal properties. Research has been intensive in antibacterial material containing various natural and inorganic substances. ${ }^{9}$ Among them, silver or silver ions have long been known to have strong inhibitory and bactericidal effects as well as a broad spectrum of antimicrobial activities. ${ }^{10,11}$ Several proposals have been developed to explain the inhibitor effects of silver ion/silver metal on bacteria. It is generally believed that heavy metals react with proteins by combining the thiol ( $\mathrm{SH}$ ) groups, which leads to the inactivation of the proteins. ${ }^{12}$ Recent, microbiological and chemical experiments implied that interaction of silver ion with thiol groups played an essential role in bacterial inactivation. ${ }^{13}$ Also, it is revealed that bulk silver in an oxygen-charged aqueous media catalyzes the complete destructive oxidation of microorganisms. ${ }^{14}$ Metal nanoparticles (Me-NPs), which have a high specific surface area and a high fraction of surface atoms have been studied extensively because of their unique physico- chemical characteristics including catalytic activity, optical properties, electronic properties, antimicrobial activity, and magnetic properties. ${ }^{15-17}$ Among Me-NPs, silver nanoparticles (AgNPs) have been known to have inhibitory and bactericidal effects. ${ }^{18}$ It can be expected that the high specific surface area and high fraction of surface atoms of Ag-NPs will lead to high antimicrobial activity as compared with bulk silver metal. ${ }^{18}$ The combined effects of Ag-NPs with the antibacterial activity of antibiotics have not been studied. The ability of pathogenic bacteria to resist antimicrobial agents has emerged in recent years and is a major health problem. ${ }^{19,20}$

Pyridazines are an important class of heterocycles, which have been the subject of extensive research, particularly in the pharmaceutical and agrochemical areas and their synthesis and applications have been comprehensively reviewed. ${ }^{21-23}$ Pyridazines including mono- and bicyclic derivatives have attracted much attention due to their potential biological activities. ${ }^{24}$ The recent advances in combinatorial chemistry and high throughput screening have confirmed the tremendous importance of heterocycles as templates in the search for novel pharmacologically useful low molecular weight compounds. ${ }^{25,26}$ Heterocycles bearing nitrogen, sulfur and thiazole moieties constitute the core structure of a number of biologically interesting compounds. ${ }^{27}$

In continuation of our research for the synthesis of series of new biologically active heterocycles, ${ }^{28-31}$ the purpose of this study was to design and synthesis new heterocycles using hydrazine pyridazine derivatives 1a-d as key materials and investigate the preliminary screening for antibacterial and antifungal activities for some of the new heterocycles using silver nanoparticles (Ag-NPs) to study the combination effect of silver nanoparticles solution on their biological activity.

\section{Results and Discussion}

Previously, we reported the synthesis of 1-[4-(2-methoxy- 
benzyl)-6-aryl-pyridazin-3(2H)-ylidene] hydrazines $\mathbf{1 a - d}^{32}$ (Scheme 1).

Treatment of 1a-d with 4-nitrobenzaldehyde in ethanol afforded the corresponding hydrazones 2a-d. Assignment of the products 2a-d were based on elemental analysis, mass spectra and IR spectra. The IR spectra showed absorption bands at 3180 $3182 \mathrm{~cm}^{-1}$ and $1595-1608 \mathrm{~cm}^{-1}$ attributed to $\mathrm{NH}$ and $\mathrm{C}=\mathrm{N}$ functions respectively and mass spectrum of $2 \mathbf{a} m / z=441\left(\mathrm{M}^{+}+2\right.$, 3.95), $440\left(\mathrm{M}^{+}+1,24.23\right), 439\left(\mathrm{M}^{+}, 93.52\right)$.

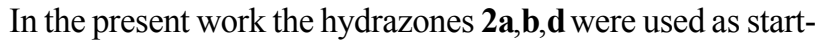
ing material for synthesis of thiazolidine or their tautomeric derivatives $\mathbf{3 a}, \mathbf{b}, \mathbf{d}$ via solid phase reaction of $\mathbf{2} \mathbf{a}, \mathbf{b}, \mathbf{d}$ with mercapto acetic acid above its melting point. The structures of the new thiazolidines were established from mass spectra, IR and ${ }^{1} \mathrm{H}$ NMR spectra. Its ${ }^{1} \mathrm{HNMR}$ spectra revealed signals at $\delta 3.81(\mathrm{~s}$, $\left.3 \mathrm{H}, \mathrm{OCH}_{3}\right), 4.37$ (s, 2H, $\left.\mathrm{CH}_{2}\right), 6.87-6.92(\mathrm{~s}, 3 \mathrm{H}, 3 \mathrm{CH}$ hetero), 7.02- $8.01(\mathrm{~m}, 13 \mathrm{H}, 3 \mathrm{Ar}-\mathrm{H}), 8.40(\mathrm{~s}, 1 \mathrm{H}, \mathrm{NH})$ and $9.80(\mathrm{~s}, 1 \mathrm{H}$, $\mathrm{OH})$.

Reduction of compounds 2a-c using $\mathrm{Zn}$ (dust) in a mixture of acetic acid and acetic anhydride $(1: 1)$ yielded 4a-c. The IR absorption spectra of $\mathbf{4 a - c}$ showed bands at $1597-1600,1693$ - 1701, 2924 - 2926 and $3172-3246 \mathrm{~cm}^{-1}$ due to $\mathrm{C}=\mathrm{N}, \mathrm{C}=\mathrm{O}$,

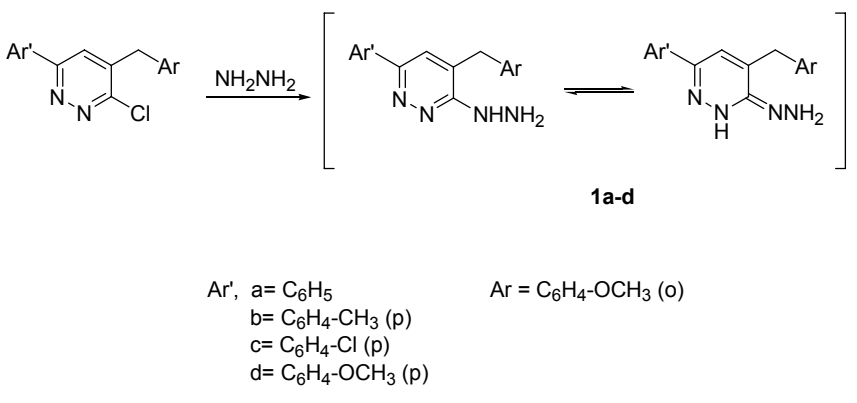

Scheme 1
$\mathrm{CH}$ (aliphatic) and NH groups. The mass spectrum of $\mathbf{4 a} \mathrm{m} / \mathrm{z}=$ $450\left(\mathrm{M}^{+}-3,1.19\right), 418(100), 375$ (3.23), 315 (8.44), 273 (12.37), 230 (6.68), 189 (3.87), 128 (11.90), 77 (22.01).

Refluxing of 1a-d with unsaturated aliphatic aldehyde such as crotonaldehyde in ethanol gave the corresponding hydrazones 5a-d. The structures of 5a-d were inferred from their elemental analysis and spectral data. Its IR spectra of 5a-d displayed bands at $1533-1544(\mathrm{C}=\mathrm{C}), 1596-1603(\mathrm{C}=\mathrm{N}), 2905$ 2911 (CH aliphatic) and $3171-3199 \mathrm{~cm}^{-1}(\mathrm{NH})$ respectively (Scheme 2).

In a routine in vitro screening for cytotoxic activity, phthalazine showed significant tumor cell growth inhibition. ${ }^{33}$ In this study, we synthesized fused heterocyclic compound such as phthalazine derivative 7 from solid phase reaction of 1c with 2-benzoyl benzoic acid. The structure of the phthalazine derivative 7 was confirmed from its elemental analysis and spectral data. The ${ }^{13} \mathrm{CNMR}$ spectrum of 7 showed signals at $\delta 127.61$, $127.61,129.41,130.46,131.45,132.30,134.88$ and 167.84 .

Reaction of 3-hydrazinopyridazine derivatives 1a-d with compounds containing active methylene group was studied. Thus 1a-d reacted with acetyl acetone to yield the corresponding pyrazolo derivatives 8a-d. The structures of product 8a-d, were confirmed by the microanalytical and spectral data. Its ${ }^{1} \mathrm{HNMR}$ spectra displayed signals at $\delta 2.22-2.36\left(\mathrm{~s}, 6 \mathrm{H}, 2 \mathrm{CH}_{3}\right), 3.82(\mathrm{~s}$, $\left.3 \mathrm{H}, \mathrm{OCH}_{3}\right), 4.14\left(\mathrm{~s}, 2 \mathrm{H}, \mathrm{CH}_{2}\right), 6.04-6.84$ (s, 2H, 2CH hetero) and 7.26-8.02 (m, 9H, 2Ar-H) (Scheme 3).

Tetrazolo pyridazines are known to undergo tautumeric transformation, the tetrazole ring can break to form the azide group, or vice versa. ${ }^{34}$ While the azide-tetrazolo tautomerism is relevant to a group of medicinal drugs, where the azide group is responsible for the mechanism of the drug activity. ${ }^{35}$ Thus in the present study tetrazolo derivatives 9 a-d were achieved by the cyclization of 3-hydrazino pyridazine derivatives 1a-d using nitrous acid which underwent tautomeric transformation via the tetrazole ring opening to form the azide group or vice versa.

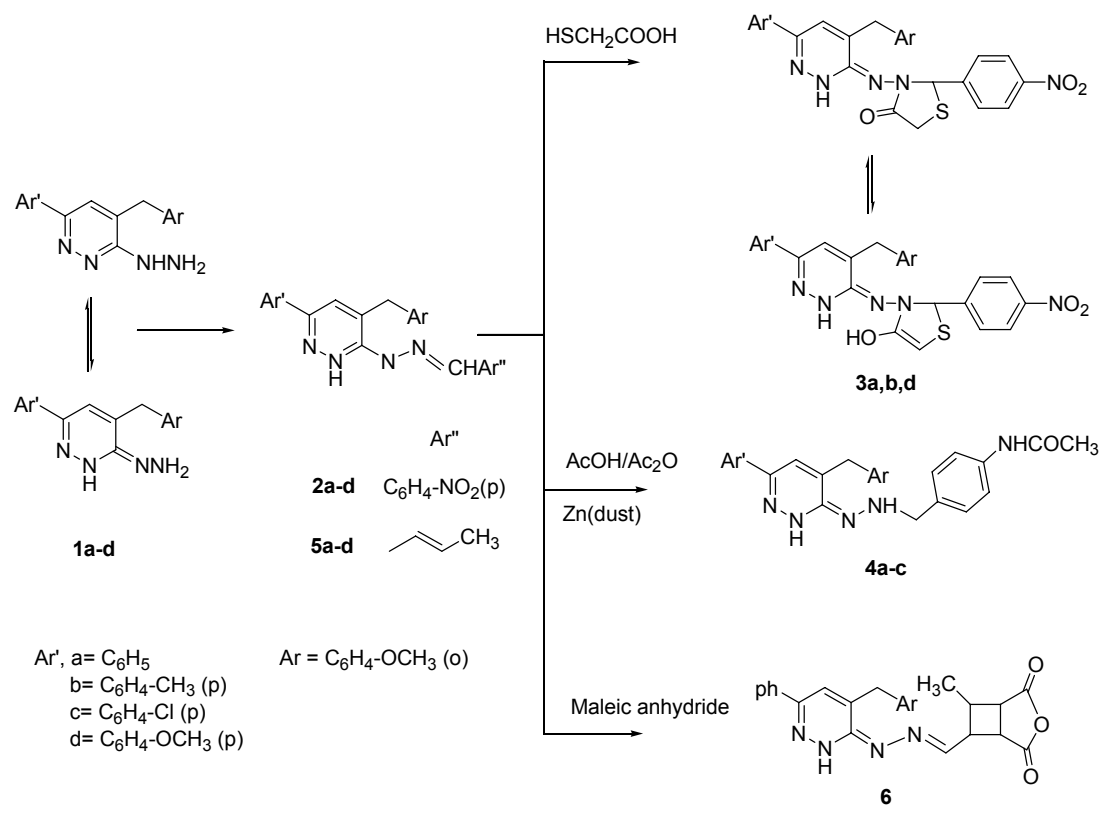

Scheme 2 


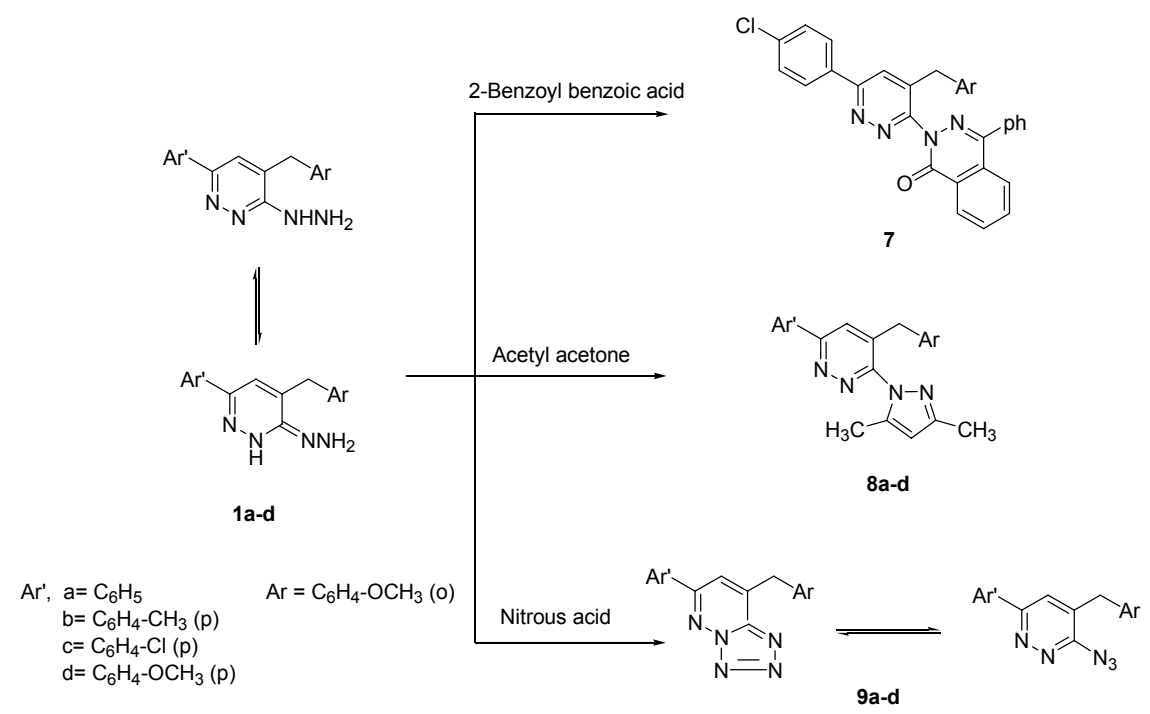

Scheme 3

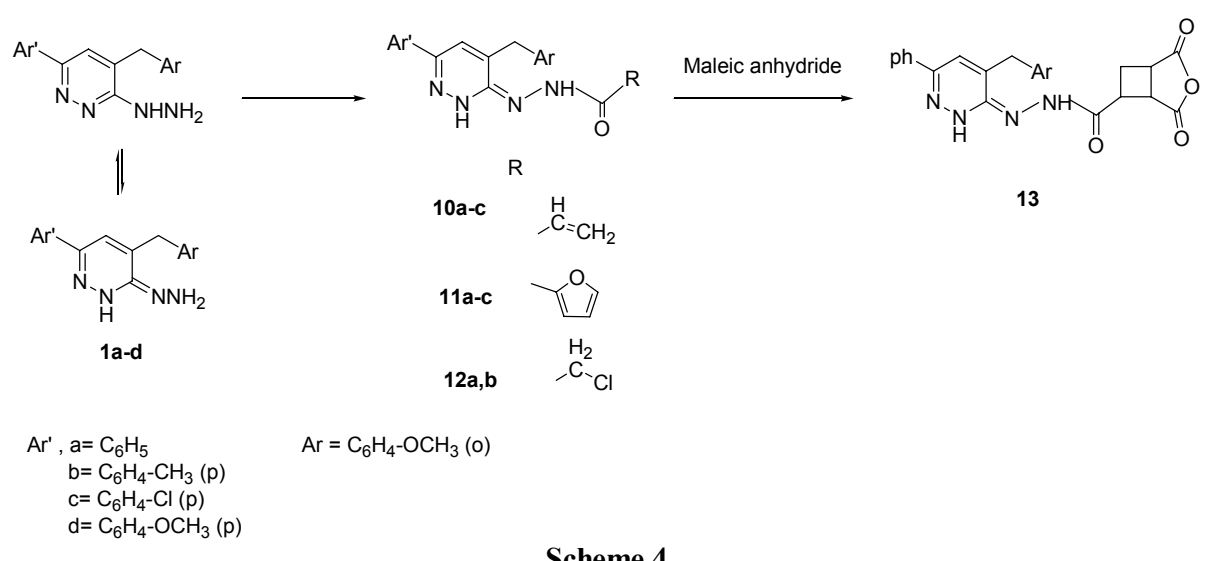

The structures of 9a-d were established other than elemental analysis and mass spectra, from IR absorption spectra which showed bands at $1164-1189$ (tetrazole), $1567-1574(\mathrm{~N}=\mathrm{N})$, $1595-1602(\mathrm{C}=\mathrm{N})$ and $2034-2077 \mathrm{~cm}^{-1}\left(\mathrm{~N}_{3}\right.$ azide $)$ respectively and the mass spectrum of $9 \mathrm{c} m / z=354\left(\mathrm{M}^{+}+3,8.19\right)$, $353\left(\mathrm{M}^{+}+2,27.27\right), 352\left(\mathrm{M}^{+}+1,18.47\right)$ (Scheme 3).

Finally, we report a convenient and versatile synthetic approach to new hydrazide derivatives 10a-d, 11a-c and 12a,b via the reaction of 1a-d with acroylchloride, 2-furoyl chloride and chloroacetyl chloride respectively. Structures of 10a-c were proved by IR spectra which showed stretching frequencies at $1537-1559(\mathrm{C}=\mathrm{C}), 1595-1603(\mathrm{C}=\mathrm{N}), 1661-1672(\mathrm{C}=\mathrm{O})$, 2929, (CH aliphatic) and $3161-3314 \mathrm{~cm}^{-1}(\mathrm{NH})$, respectively. The presence of the double bond $(\mathrm{C}=\mathrm{C})$ in compounds $\mathbf{5} \mathbf{a}$ and 10a were confirmed chemically from the Enone reaction of products 5a and 10a with maleic anhydride to give the expected products 6,13 . The structures of 6,13 were established other than elemental analysis and mass spectra. Their IR spectra showed bands at $1600-1601(\mathrm{C}=\mathrm{N}), 1650-1655 \& 1727-1729$ $(\mathrm{C}=\mathrm{O})$ and $3400-3421 \mathrm{~cm}^{-1}(\mathrm{NH})$. The mass spectrum of 13 $m / z=457\left(\mathrm{M}^{+}-1,6.73\right)$ (Scheme 4).

Characterization of Ag-NPs solution. Silver nanoparticles

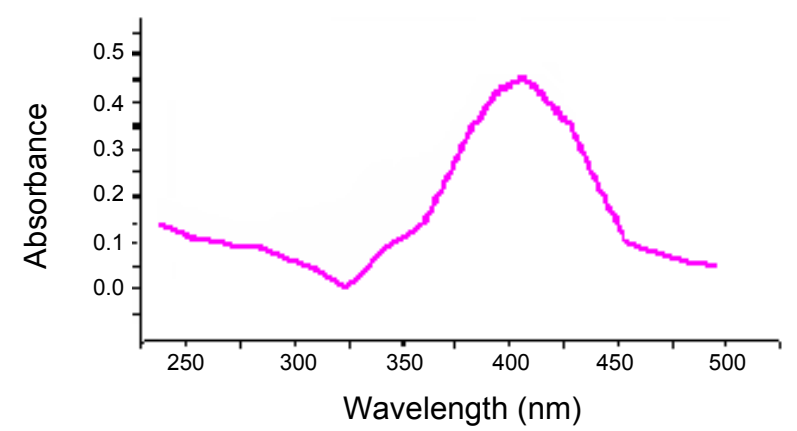

Figure 1. UV-visible spectra for Ag-NPs solution, which showed $\lambda_{\max }$ at 400 .

solution was prepared using simple methodology by chemical reduction method reported, ${ }^{36}$ using solution of $\mathrm{AgNO}_{3}$ and trisodium citrate was added with heating under magnetic stirring, then the solution turned to yellow colour.

To confirm the formation of silver nanoparticles in this solution, we carried out an UV-visible absorption study and TEM imaging. In Fig. 1, a strong characteristic absorption peak around $400 \mathrm{~nm}$ is noted for the silver nanoparticles in the solution due 
to the surface plasmon resonance effect. Observation of this strong but broad surface plasmon peak has been well documented for various Ag-NPs size. . $^{37-39}$

Transmission electron microscopy (TEM) images of the AgNPs solution which showed different size of Ag-NPs (Figure 2)

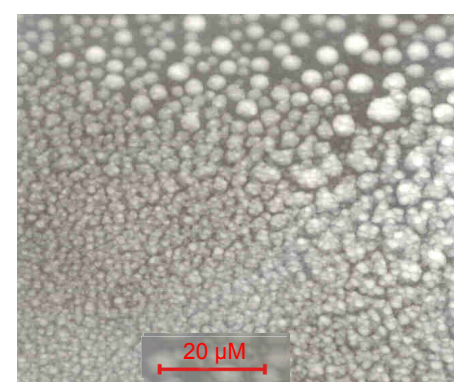

Figure 2. TEM micrograph of Ag-NPs solution. Scale bar $=20 \mu \mathrm{m}$.

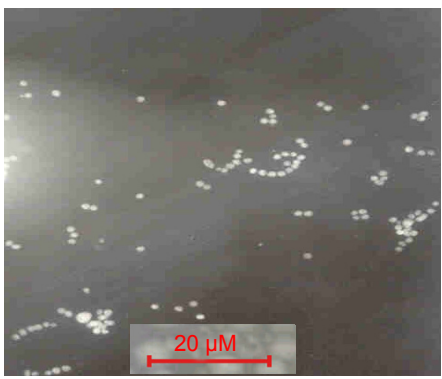

Figure 3. TEM micrograph of $\mathbf{4 a}$ after addition of Ag-NPs solution. Scale bar $=20 \mu \mathrm{m}$

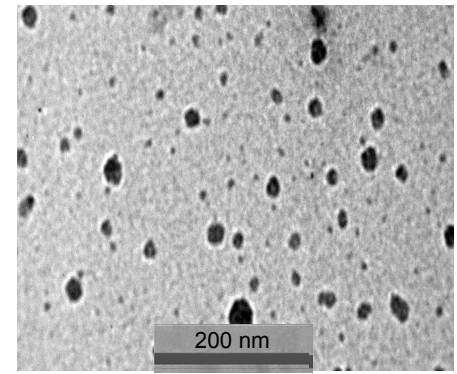

Figure 4. TEM micrograph of $\mathbf{8 b}$ after addition of Ag-NPs solution. Scale bar $=200 \mathrm{~nm}$ were recorded using a Zeiss Electron Microscope 10, operating at power $60 \mathrm{kV}$. TEM samples were prepared by dispersing 2 - 3 drops of Ag-NPs solution on copper grid and dried at room temperature after removal of excess solution using a filter paper.

A solution of compounds $4 \mathbf{a}, 8 \mathbf{a}, \mathbf{b}, 10 \mathrm{a}, 11 \mathbf{b}$ and $\mathbf{1 2 b}$ were stirred with Ag-NPs solution, the residue products obtained in nano form were confirmed by (TEM) which showed different size of nano particles (Figures 3-6).

Microbial experimentation. Microbial investigations were done to find the effect of some newly synthesized compounds against (Gram +ve) Staphylococcus aureus ATCC (12600) and (Gram -ve) bacteria Escherichia coli ATCC (11775), in addition to their antifungal activity against Aspergillus flavus and Candida albicans with or without silver nanoparticles (Ag-NPs) solution. The preliminary studies of the biological assay were performed according to the agar diffusion method ${ }^{40-43}$ at a concentration $(20 \mathrm{mg} / \mathrm{mL})$ using $\mathrm{CHCl}_{3}$ or DMSO as solvents. The

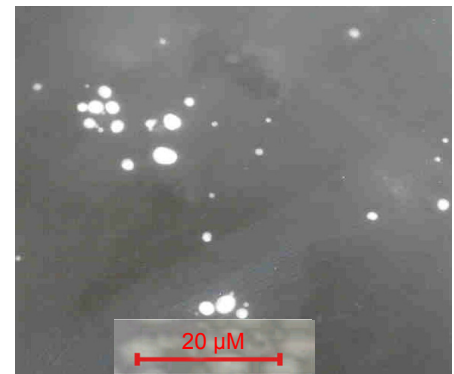

Figure 5. TEM micrograph of 10a after addition of Ag-NPs solution. Scale bar $=20 \mu \mathrm{m}$

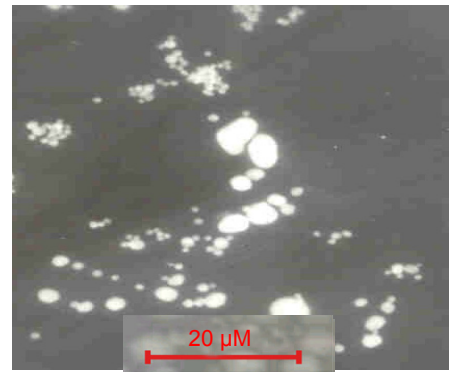

Figure 6. TEM micrograph of $\mathbf{1 1 b}$ after addition of Ag-NPs solution. Scale bar $=20 \mu \mathrm{m}$

Table 1. Inhibition Zone diameter ( $\mathrm{mm} / \mathrm{mg}$ Sample) of some compounds against $S$. aureus \& E. coli

\begin{tabular}{|c|c|c|c|c|c|c|}
\hline \multirow[b]{2}{*}{ Comp. } & \multicolumn{3}{|c|}{ Staphylococcus aureus } & \multicolumn{3}{|c|}{ Escherichia coli } \\
\hline & Without Ag-NPs (a) & With Ag-NPs (b) & $\begin{array}{c}\text { Fold increase } \%= \\
((b-a) / a) \times 100\end{array}$ & Without Ag-NPs (a) & With Ag-NPs (b) & $\begin{array}{c}\text { Fold increase } \%= \\
((b-a) / a) \times 100\end{array}$ \\
\hline $\begin{array}{c}\text { Tetracyclin } \\
\text { Antibacterial agent } \\
\text { (as standard) }\end{array}$ & 20 & & & 22 & & \\
\hline $4 a$ & 13 & 13 & 0.0 & 12 & 12 & 0.0 \\
\hline $8 a$ & 14 & 14 & 0.0 & 14 & 15 & 7.14 \\
\hline $\mathbf{8 b}$ & 15 & 22 & 46.66 & 16 & 20 & 25 \\
\hline $10 \mathrm{a}$ & 15 & 16 & 6.66 & 14 & 15 & 7.14 \\
\hline 11b & 12 & 14 & 16.66 & 12 & 14 & 16.66 \\
\hline $12 b$ & 12 & 14 & 16.66 & 12 & 12 & 0.0 \\
\hline
\end{tabular}


Table 2. Inhibition Zone diameter (mm/mg Sample) of some compounds against Asp. flavus \& Can. albians

\begin{tabular}{|c|c|c|c|c|c|}
\hline \multirow[b]{2}{*}{ Comp. } & \multicolumn{2}{|c|}{ Aspergillus flavus } & \multicolumn{3}{|c|}{ Candida albicans } \\
\hline & Without Ag-NPs & With Ag-NPs & Withou Ag-NPs (a) & With Ag-NPs (b) & $\begin{array}{c}\text { Fold increase } \%= \\
((b-a) / a) \times 100\end{array}$ \\
\hline Amphotericin B & \multirow{2}{*}{\multicolumn{2}{|c|}{20}} & \multirow{2}{*}{\multicolumn{2}{|c|}{20}} & \\
\hline $\begin{array}{l}\text { Antifungal agent } \\
\text { (as standard) }\end{array}$ & & & & & \\
\hline $\mathbf{8 b}$ & 0.0 & 0.0 & 12 & 15 & 25 \\
\hline $9 c$ & 0.0 & 0.0 & 13 & 13 & 0.0 \\
\hline $10 \mathrm{a}$ & 0.0 & 14 & 13 & 14 & 7.69 \\
\hline $11 b$ & 0.0 & 0.0 & 12 & 13 & 8.33 \\
\hline $12 b$ & 0.0 & 0.0 & 13 & 13 & 0.0 \\
\hline
\end{tabular}

results of the in vitro antimicrobial activity were recorded as average diameter of inhibition zone in $\mathrm{mm}$, are given in Table $1,2$.

Antibacterial activity. The combination effect of silver nanoparticles (Ag-NPs) solution with different compounds was investigated against Staphylococcus aureus and Escherichia coli using the diffusion method. ${ }^{40-43}$ The diameter of inhibition zones (in millimeters) around the different compounds disks with or without Ag-NPs against test strains are shown in Table 1.

The antibacterial activities of $\mathbf{8 b}, \mathbf{1 0 a}$ and $\mathbf{1 1 b}$ increased in the presence of Ag-NPs solution against both test strains. No enhancing effect of $\mathbf{4 a}$ on the antibacterial activities against Staphylococcus aureus and Escherichia coli. The highest fold increases in area were observed for $\mathbf{8 b}, \mathbf{1 0 a}$ and $\mathbf{1 2 b}$ in presence of Ag-NPs against Staphylococcus aureus. In contrast, the highest fold increase in area was observed for $\mathbf{8 b}$ against Escherichia coli.

Antifungal activity. The antifungal activities of 10a increased in presence of Ag-NPs solution against Aspergillus flavus and Candida albicans. No enhancing effect on the antifungal activities of $9 \mathbf{c}$ and $\mathbf{1 2 b}$ in presence of Ag-NPs against Aspergillus flavus and Candida albicans was observed. The highest fold increases in area were observed for $\mathbf{8 b}$ and $\mathbf{1 1 b}$ in presence of Ag-NPs solution against Candida albicans, but they had no effect against Aspergillus flavus.

\section{Conclusion}

The current study involved the design and synthesis of new heterocycles based on pyridazin moiety using simple synthetic route to evaluate their antimicrobial and antifungal activities, and study the effect of silver nanoparticles solution on their biological activities. The study showed that the antimicrobial activity of compound $\mathbf{8 b}$ was the highest enhancing effect in the presence of silver nanoparticles solution against Staphylococcus aureus and Escherichia coli. Compound 10a exhibited the strongest enhancing effect of silver nanoparticles solution against Aspergillus flavus and Candida albicans. Compound $\mathbf{8 b}$ exhibited the strongest enhancing effect of silver nanoparticles solution against Candida albicans.

\section{Experimental}

General. Melting points are uncorrected. The IR spectra of the compounds were recorded on a Perkin-Elmer spectrophotometer model 1430 as potassium bromide pellets and frequen- cies are reported in $\mathrm{cm}^{-1}$. The ${ }^{1} \mathrm{HNMR}$ spectra were observed on Varian Genini-300 MHz spectrometer and chemical shifts ( ) are in ppm. The mass spectra were recorded on a mass spectrometer HP model MS -QPL000EX (Shimadzu) at $70 \mathrm{eV}$. Elemental analysis and antimicrobial activity evaluations were carried out at the Microanalytical Center of Cairo University, Giza, Egypt. The TEM were recorded on a Zeiss EM 10 (West Germany) with power $60 \mathrm{kV}$ at National Research Center.

General procedure for the preparation of hydrazones $2 \mathrm{a}-\mathrm{d} \&$ 5a-d from 1-[4-(2-methoxy benzyl)-6-arylpyridazin-3(2H)ylidene] hydrazines 1a-d and different aldehydes. A solution of compounds 1a-d $(1 \mathrm{mmol})$ in ethanol $(20 \mathrm{~mL})$ was treated with the corresponding aldehydes $(1 \mathrm{mmol})$. The reaction mixture was refluxed for $5 \mathrm{hrs}$. The separated solid was filtered off and crystallized from the appropriate solvent to afford the title compounds $\mathbf{2 a - d} \& \mathbf{5 a - d}$.

1-[4-(2-Methoxybenzyl)-6-phenylpyridazin-3(2H)-ylidene]2-(4-nitrobenzylidene) hydrazine (2a): Prepared from 4-nitrobenzaldehyde, yellow crystals in $90 \%$ yield, mp $232-4{ }^{\circ} \mathrm{C}$ (benzene); Mass spectrum $m / z 441\left(\mathrm{M}^{+}+2,3.95\right), 440\left(\mathrm{M}^{+}+1\right.$, 24.23), $439\left(\mathrm{M}^{+}, 93.52\right)$, IR: $1590(\mathrm{C}=\mathrm{N})$ and $3181 \mathrm{~cm}^{-1}(\mathrm{NH})$. Anal. Calcd for $\mathrm{C}_{25} \mathrm{H}_{21} \mathrm{~N}_{5} \mathrm{O}_{3}: \mathrm{C}, 68.33 ; \mathrm{H}, 4.82 ; \mathrm{N}, 15.94 \%$. Found: C, 67.92; H, 5.30; N, 15.77\%.

1-[4-(2-Methoxybenzyl)-6-methylphenylpyridazin-3(2H)ylidene]-2-(4-nitrobenzylidene) hydrazine (2b): Prepared from 4-nitrobenzaldehyde, yellow crystals in $90 \%$ yield, mp $227-9^{\circ} \mathrm{C}$ (benzene); Mass spectrum $m / z 455\left(\mathrm{M}^{+}+2,5.13\right), 454\left(\mathrm{M}^{+}+1\right.$, 29), $453\left(\mathrm{M}^{+}, 99.29\right)$, IR: $1593(\mathrm{C}=\mathrm{N})$ and $3182 \mathrm{~cm}^{-1}(\mathrm{NH})$. Anal. Calcd for $\mathrm{C}_{26} \mathrm{H}_{23} \mathrm{~N}_{5} \mathrm{O}_{3}$ : C, 68.86; H, 5.11; N, 15.44\%. Found: C, 68.53; H, 5.48; N, 15.48\%.

1-[4-(2-Methoxybenzyl)-6-chlorophenylpyridazin-3(2H)ylidene]-2-(4-nitrobenzylidene) hydrazine (2c): Prepared from 4-nitrobenzaldehyde, yellow crystals in $90 \%$ yield, $\mathrm{mp} 245-7^{\circ} \mathrm{C}$ (benzene); Mass spectrum $m / z 476\left(\mathrm{M}^{+}+3,7.01\right), 475\left(\mathrm{M}^{+}+2\right.$, 29.22), $474\left(\mathrm{M}^{+}+1,27.41\right), 473\left(\mathrm{M}^{+}, 74.73\right), \mathrm{IR}: 1594(\mathrm{C}=\mathrm{N})$ and $3179 \mathrm{~cm}^{-1}(\mathrm{NH}) .{ }^{1} \mathrm{HNMR}$ (DMSO, $\left.200 \mathrm{MHz}\right) \delta 3.90(\mathrm{~s}, 3 \mathrm{H}$, $\left.\mathrm{OCH}_{3}\right), 4.22\left(\mathrm{~s}, 2 \mathrm{H}, \mathrm{CH}_{2}\right), 6.89(\mathrm{~s}, 1 \mathrm{H}, \mathrm{CH}$ hetero $), 7.56-7.72(\mathrm{~m}$, $12 \mathrm{H}, 3 \mathrm{Ar}-\mathrm{H}), 8.27(\mathrm{~s}, 1 \mathrm{H}, \mathrm{NH})$ and $11.42(\mathrm{~s}, 1 \mathrm{H}, \mathrm{N}=\mathrm{CH})$ Anal. Calcd for $\mathrm{C}_{25} \mathrm{H}_{20} \mathrm{~N}_{5} \mathrm{O}_{3} \mathrm{Cl}$ : C, 63.36; H, 4.25; N, 14.78\%; Found: C, 63.06; H, 4.07; N, 14.53\%.

1-[4-(2-Methoxybenzyl)-6-methoxyphenylpyridazin-3(2H)ylidene]-2-(4-nitrobenzylidene) hydrazine (2d): Prepared from 4-nitrobenzaldehyde, yellow crystals in 90\% yield, mp 224 - 6 ${ }^{\circ} \mathrm{C}$ (benzene); Mass spectrum $m / z 471\left(\mathrm{M}^{+}+2,2.91\right), 470\left(\mathrm{M}^{+}+1\right.$, 17.76), $469\left(\mathrm{M}^{+}, 60.90\right)$, IR: $1607(\mathrm{C}=\mathrm{N})$ and $3180 \mathrm{~cm}^{-1}(\mathrm{NH})$. 
Anal. Calcd for $\mathrm{C}_{26} \mathrm{H}_{23} \mathrm{~N}_{5} \mathrm{O}_{4}: \mathrm{C}, 66.51 ; \mathrm{H}, 4.94 ; \mathrm{N}, 14.92 \%$. Found: C, 67.03; H, 5.37; N, 14.80\%.

1-[4-(2-Methoxybenzyl)-6-phenylpyridazin-3(2H)-ylidene]2-((E)butan-2-en-1-ylidene) hydrazine (5a): Prepared from crotonaldehyde, yellow crystals in $92.6 \%$ yield, mp $146-8^{\circ} \mathrm{C}$ (ethanol); Mass spectrum $m / z 359\left(\mathrm{M}^{+}+1,5.53\right), 358\left(\mathrm{M}^{+}, 22.36\right)$, IR: $1538(\mathrm{C}=\mathrm{C}), 1596(\mathrm{C}=\mathrm{N}), 2910(\mathrm{CH}$ aliphatic $)$ and 3199 $\mathrm{cm}^{-1}(\mathrm{NH}),{ }^{1} \mathrm{HNMR}\left(\mathrm{CDCl}_{3}, 300 \mathrm{MHz}\right) \delta 1.91-1.94(\mathrm{~d}, 3 \mathrm{H}$, $\left.\mathrm{CH}_{3}\right), 3.81\left(\mathrm{~s}, 3 \mathrm{H}, \mathrm{OCH}_{3}\right), 3.92\left(\mathrm{~s}, 2 \mathrm{H}, \mathrm{CH}_{2}\right), 6.15-6.22(\mathrm{q}, 1 \mathrm{H}$, $\mathrm{CH}), 6.35-6.44(\mathrm{t}, 1 \mathrm{H}, \mathrm{CH}), 6.71(\mathrm{~s}, 1 \mathrm{H}, \mathrm{CH}$ hetero), 6.93-7.35 $(\mathrm{m}, 9 \mathrm{H}, 2 \mathrm{Ar}-\mathrm{H}), 7.58(\mathrm{~s}, 1 \mathrm{H}, \mathrm{NH})$ and $8.13-8.16(\mathrm{~d}, 1 \mathrm{H}, \mathrm{N}=\mathrm{CH})$. Anal.Calcd for $\mathrm{C}_{22} \mathrm{H}_{22} \mathrm{~N}_{4} \mathrm{O}: \mathrm{C}, 73.72 ; \mathrm{H}, 6.19 ; \mathrm{N}, 15.63 \%$. Found: C, 73.51; H, 6.02; N, 15.34\%.

1-[4-(2-Methoxybenzyl)-6-methylphenylpyridazin-3(2H)ylidene]-2-((E)butan-2-en-1-ylidene)hydrazine (5b): Prepared from crotonaldehyde, yellow crystals in $80 \%$ yield, $\mathrm{mp} 150{ }^{\circ} \mathrm{C}$ (ethanol); Mass spectrum $m / z 373\left(\mathrm{M}^{+}+1,6.42\right), 372\left(\mathrm{M}^{+}, 24.63\right)$, IR: $1541(\mathrm{C}=\mathrm{C}), 1599(\mathrm{C}=\mathrm{N}), 2911(\mathrm{CH}$ aliphatic $)$ and 3176 $\mathrm{cm}^{-1}(\mathrm{NH})$. Anal. Calcd for $\mathrm{C}_{23} \mathrm{H}_{24} \mathrm{~N}_{4} \mathrm{O}: \mathrm{C}, 74.17 ; \mathrm{H}, 6.49 ; \mathrm{N}$, $15.04 \%$. Found: C, 73.81; H, 6.02; N, 14.75\%.

1-[4-(2-Methoxybenzyl)-6-chlorophenylpyridazin-3(2H)ylidene]-2-((E)butan-2-en-1-ylidene) hydrazine (5c): Prepared from crotonaldehyde, yellow crystals in $70 \%$ yield, mp $182{ }^{\circ} \mathrm{C}$ (ethanol); Mass spectrum $m / z 395\left(\mathrm{M}^{+}+3,1.43\right), 394\left(\mathrm{M}^{+}+2\right.$, 7.39), $393\left(\mathrm{M}^{+}+1,9.93\right), 392\left(\mathrm{M}^{+}, 22.18\right)$, IR: $1533(\mathrm{C}=\mathrm{C}), 1596$ $(\mathrm{C}=\mathrm{N}), 2905$ (CH aliphatic) and $3171 \mathrm{~cm}^{-1}(\mathrm{NH})$; Anal. Calcd for $\mathrm{C}_{22} \mathrm{H}_{21} \mathrm{~N}_{4} \mathrm{OCl}$ : C, 67.26; H, 5.39; N, 14.26; Cl, 9.02\%. Found: C, 67.40; H, 5.44; N, 14.28; Cl, 9.12\%.

1-[4-(2-Methoxybenzyl)-6-methoxyphenylpyridazin-3(2H)ylidene]-2-((E)butan-2-en-1-ylidene) hydrazine (5d): Prepared from crotonaldehyde, yellow crystals in $80 \%$ yield, mp 126 $8{ }^{\circ} \mathrm{C}$ (ethanol); Mass spectrum $\mathrm{m} / z 388\left(\mathrm{M}^{+}, 0.96\right)$, IR: 1544 $(\mathrm{C}=\mathrm{C}), 1603(\mathrm{C}=\mathrm{N}), 2909(\mathrm{CH}$ aliphatic $)$ and $3176 \mathrm{~cm}^{-1}(\mathrm{NH})$; ${ }^{1} \mathrm{HNMR}\left(\mathrm{CDCl}_{3}, 300 \mathrm{MHz}\right) \delta 1.90-1.93\left(\mathrm{~d}, 3 \mathrm{H}, \mathrm{CH}_{3}\right), 3.74-3.82$ $\left(\mathrm{s}, 6 \mathrm{H}, 2 \mathrm{OCH}_{3}\right), 3.92\left(\mathrm{~s}, 2 \mathrm{H}, \mathrm{CH}_{2}\right), 6.12-6.19(\mathrm{q}, 1 \mathrm{H}, \mathrm{CH})$, 6.34-6.43 (t, 1H, CH), $6.69(\mathrm{~s}, 1 \mathrm{H}, \mathrm{CH}$ hetero $), 6.88-7.55(\mathrm{~m}$, $8 \mathrm{H}, 2 \mathrm{Ar}-\mathrm{H}), 7.82(\mathrm{~s}, 1 \mathrm{H}, \mathrm{NH})$ and $8.40(\mathrm{~d}, 1 \mathrm{H}, \mathrm{N}=\mathrm{CH})$. Anal. Calcd for $\mathrm{C}_{23} \mathrm{H}_{24} \mathrm{~N}_{4} \mathrm{O}_{2}$ : C, 71.11; H, 6.23; $\mathrm{N}, 14.42 \%$. Found: C, 70.80; H, 6.26; N, 14.23\%.

Reaction of hydrazone derivatives $2 \mathrm{a}, \mathrm{b}, \mathrm{d}$ with mercapto acetic acid. The hydrazone derivatives $\mathbf{2 a}, \mathbf{b}, \mathbf{d}(1 \mathrm{mmol})$ was heated above its melting point with mercapto acetic acid $(1 \mathrm{mmol})$ in sand bath for $3 \mathrm{hrs}$. The product which solidified on cooling was triturated with pet. ether (bp $40-60^{\circ} \mathrm{C}$ ), filtered off and crystallized from the proper solvent.

3-[4-(2-Methoxybenzyl)-6-phenylpyridazin-3(2H)-ylideneamino]-2-(4-nitrophenyl)-thiazolidin-4-one (3a): Brown crystals in $40 \%$ yield, $\mathrm{mp}>300{ }^{\circ} \mathrm{C}$ (DMF); Mass spectrum $\mathrm{m} / \mathrm{z} 467$ $\left(\mathrm{M}^{+}-46,11.36\right), 439$ (30.60), 368 (25.55), 339 (12.30), 313 (17.82), 284 (35.33), 271 (10.88), 257 (69.87), 239 (19.56), 213 (48.26), 199 (23.50), 183 (76.50), 157 (22.08), 129 (90.06), 73 (100), 60 (84.86), 55 (32.65), IR: $1317 \& 1466\left(\mathrm{NO}_{2}\right), 1603$ $(\mathrm{C}=\mathrm{N}), 4.37\left(\mathrm{~s}, 2 \mathrm{H}, \mathrm{CH}_{2}\right), 3215(\mathrm{NH})$ and $3356 \mathrm{~cm}^{-1}(\mathrm{OH})$. ${ }^{1} \mathrm{HNMR}(\mathrm{DMSO}, 300 \mathrm{MHz}) \delta 3.81\left(\mathrm{~s}, 3 \mathrm{H}, \mathrm{OCH}_{3}\right), 6.87-6.92(\mathrm{~s}$, $3 \mathrm{H}, 3 \mathrm{CH}$ hetero), 7.02-8.01 (m, 13H, 3Ar-H), 8.40 (s, 1H, NH) and $9.80(\mathrm{~s}, 1 \mathrm{H}, \mathrm{OH})$. Anal. Calcd for $\mathrm{C}_{27} \mathrm{H}_{23} \mathrm{~N}_{5} \mathrm{O}_{4} \mathrm{~S}: \mathrm{C}, 63.14 ; \mathrm{H}$, 4.51 ; N, 13.64; S, 6.24\%. Found: C, 63.40; H, 4.50; N, 13.65; $\mathrm{S}, 6.51 \%$.
3-[4-(2-Methoxybenzyl)-6-methylphenylpyridazin-3(2H)ylidene-amino]-2-(4-nitrophenyl)-thiazolidin-4-one (3b): Brown crystals in $34 \%$ yield, $\mathrm{mp}>300^{\circ} \mathrm{C}$ (DMF); Mass spectrum $\mathrm{m} / \mathrm{z}$ $450\left(\mathrm{M}^{+}-77,0.41\right), 437$ (0.50),407 (30.79), 392 (2.33), 329 (5.88), 313 (100), 299 (4.86), 238 (5.25), 212 (3.41), 196 (16.51), 115 (4.78), 91 (12.17), 77 (6.64), 65 (6.01), IR: $1320 \& 1464$ $\left(\mathrm{NO}_{2}\right), 1602(\mathrm{C}=\mathrm{N}), 1662(\mathrm{C}=\mathrm{O})$ and $3422 \mathrm{~cm}^{-1}$ broad band for $(\mathrm{OH}, \mathrm{NH})$, Anal. Calcd for $\mathrm{C}_{28} \mathrm{H}_{25} \mathrm{~N}_{5} \mathrm{O}_{4} \mathrm{~S}: \mathrm{C}, 63.74 ; \mathrm{H}, 4.78 ; \mathrm{N}$, 13.27; S, 6.08\%. Found: C, 64.16; H, 5.03; N, 13.18; S, 6.20\%.

3-[4-(2-Methoxybenzyl)-6-methoxyphenylpyridazin-3(2H)ylidene-amino]-2-(4-nitro phenyl)-thiazolidin-4-one (3d): Brown crystals in $30 \%$ yield, mp $204-6{ }^{\circ} \mathrm{C}$ (DMF); Mass spectrum $\mathrm{m} / \mathrm{z} 534\left(\mathrm{M}^{+}-9,4.34\right), 533$ (15.50), 449 (13.01), 407 (34.04), 313 (37.27), 239 (31.18), 185 (24.26), 149 (69.56), 57 (100), IR: $1317 \& 1464\left(\mathrm{NO}_{2}\right), 1602(\mathrm{C}=\mathrm{N}), 1682(\mathrm{C}=\mathrm{O})$ and $3316 \mathrm{~cm}^{-1}$ broad band for $(\mathrm{OH}, \mathrm{NH})$. Anal. Calcd for $\mathrm{C}_{28} \mathrm{H}_{25} \mathrm{~N}_{5} \mathrm{O}_{5} \mathrm{~S}$ : N, 12.88 ; S, 5.90\%. Found: N, 12.81; S, 5.75\%.

Reduction of hydrazone derivatives 2a-c. A solution of compounds $2 \mathbf{a}-\mathbf{c}(1 \mathrm{mmol})$ in mixture of (acetic anhydride/glacial acetic acid) (1:1) $(20 \mathrm{~mL})$ was refluxed with $\mathrm{Zn}$ (dust) $(0.5 \mathrm{~g})$ for $5 \mathrm{hrs}$. The reaction mixture was filtered on hot and poured onto ice. The solid product obtained was filtered, dried and crystallized from the appropriate solvent.

1-[4-(2-Methoxybenzyl)-6-phenylpyridazin-3(2H)-ylidene]2-(4-acetamidobenzyl) hydrazines (4a): Yellow crystals in $80 \%$ yield, mp $194-6{ }^{\circ} \mathrm{C}$ (benzene); Mass spectrum $m / z 450\left(\mathrm{M}^{+}-3\right.$, 1.19), 418 (100), 375 (3.23), 315 (8.44), 273 (12.37), 230 (6.68), 189 (3.87), 128 (11.90), 77 (22.01), IR: $1600(\mathrm{C}=\mathrm{N}), 1695$ $(\mathrm{C}=\mathrm{O}), 2926$ (CH aliphatic) and $3246 \mathrm{~cm}^{-1}(\mathrm{NH})$. Anal. Calcd for $\mathrm{C}_{27} \mathrm{H}_{27} \mathrm{~N}_{5} \mathrm{O}_{2}$ : C, 71.50; H, 6.00; N, 15.44\%. Found: C, $71.36 ; \mathrm{H}, 5.70 ; \mathrm{N}, 15.48 \%$.

1-[4-(2-Methoxybenzyl)-6-methylphenylpyridazin-3(2H)ylidene]-2-(4-acetamido benzyl)hydrazines (4b): Yellow crystals in $75 \%$ yield, $\mathrm{mp} 150{ }^{\circ} \mathrm{C}$ (benzene); Mass spectrum $\mathrm{m} / \mathrm{z} 467$ $\left(\mathrm{M}^{+}, 0.24\right)$, IR: $1600(\mathrm{C}=\mathrm{N}), 1693(\mathrm{C}=\mathrm{O}), 2924$ ( $\mathrm{CH}$ aliphatic) and $3172-3244 \mathrm{~cm}^{-1}(\mathrm{NH}) .{ }^{1} \mathrm{HNMR}\left(\mathrm{CDCl}_{3}, 300 \mathrm{MHz}\right) \delta 2.86$ $\left(\mathrm{s}, 3 \mathrm{H}, \mathrm{CH}_{3}\right), 3.83\left(\mathrm{~s}, 3 \mathrm{H}, \mathrm{OCH}_{3}\right), 4.48\left(\mathrm{~s}, 2 \mathrm{H}, \mathrm{CH}_{2}\right), 4.57(\mathrm{~s}$, $\left.3 \mathrm{H}, \mathrm{COCH}_{3}\right), 6.94\left(\mathrm{~s}, 1 \mathrm{H}, \mathrm{CH}\right.$ hetero), $6.97\left(\mathrm{~s}, 2 \mathrm{H}, \mathrm{CH}_{2}-\mathrm{N}\right)$, 7.24-7.79 (m, 12H, 3Ar-H) and 8.40-8.88 (s, 3H, 3NH), Anal. Calcd for $\mathrm{C}_{28} \mathrm{H}_{29} \mathrm{~N}_{5} \mathrm{O}_{2}: \mathrm{C}, 71.93 ; \mathrm{H}, 6.25 ; \mathrm{N}, 14.98 \%$. Found: C, 71.80; H, 6.00; N, 14.98\%.

1-[4-(2-Methoxybenzyl)-6-chlorophenylpyridazin-3(2H)ylidene]-2-(4-acetamidobenzyl) hydrazines (4c): Yellow crystals in $84 \%$ yield, $\mathrm{mp} 102-4^{\circ} \mathrm{C}$ (benzene); Mass spectrum $\mathrm{m} / \mathrm{z}$ $487.5\left(\mathrm{M}^{+}, 0.24\right)$, IR: $1597(\mathrm{C}=\mathrm{N}), 1701(\mathrm{C}=\mathrm{O}), 2926(\mathrm{CH}$ aliphatic) and $3245 \mathrm{~cm}^{-1}(\mathrm{NH})$. Anal. Calcd for $\mathrm{C}_{27} \mathrm{H}_{26} \mathrm{~N}_{5} \mathrm{O}_{2} \mathrm{Cl}$ : C, 66.46; H, 5.37; N, 14.35; Cl, 7.27\%. Found: C, 66.36; H, 5.22; $\mathrm{N}, 14.35 ; \mathrm{Cl}, 7.10 \%$.

Cyclization reaction of 1-[4-(2-methoxybenzyl)-6-arylpyridazin-3(2H)-ylidene] hydrazines 1a-d with 2-benzoyl benzoic acid or acetyl acetone. Fusion of compound 1a-d(1 mmole) with 2-benzoyl benzoic acid or acetyl acetone ( $1 \mathrm{mmole}$ ) for $3 \mathrm{hrs}$ above its melting point. The molten product obtained on cooling was triturated with pet. ether (bp $40-60{ }^{\circ} \mathrm{C}$ ) and crystallized from the proper solvent.

2-[4-(2-Methoxybenzyl)-6-chlorophenylpyridazin-3-yl]-4phenylphthalazin-1(2H)one (7). Prepared from 2-benzoyl benzoic acid, brown crystals in $65 \%$ yield , mp $97-8^{\circ} \mathrm{C}$ (benzene); 
Mass spectrum $m / z 530\left(\mathrm{M}^{+}, 0.27\right)$, IR: $1597(\mathrm{C}=\mathrm{N})$ and 1662 $\mathrm{cm}^{-1}(\mathrm{C}=\mathrm{O}),{ }^{1} \mathrm{HNMR}\left(\mathrm{CDCl}_{3}, 300 \mathrm{MHz}\right) \delta 3.80\left(\mathrm{~s}, 3 \mathrm{H}, \mathrm{OCH}_{3}\right)$, $4.42\left(\mathrm{~s}, 2 \mathrm{H}, \mathrm{CH}_{2}\right), 6.90(\mathrm{~s}, 1 \mathrm{H}, \mathrm{CH}$ hetero) and 7.03-7.97 (m, $17 \mathrm{H}, 4 \mathrm{Ar}-\mathrm{H}) .{ }^{13} \mathrm{CNMR}$ (DMSO) $\delta 29.64,55.27,111.03,120.43$, $122.81,123.12$, 124.94, 126.69, 127.61, 127.61, 128.92, 128.92, $128.92,128.92,129.20,129.20,129.41,129.41,129.41,130.26$, $130.46,131.45,131.45,131.45,132.30,132.93,134.21,134.88$, $149.79,158.29,160.59$ and 167.84 , Anal. Calcd for $\mathrm{C}_{32} \mathrm{H}_{23}$ $\mathrm{N}_{4} \mathrm{O}_{2} \mathrm{Cl}$ : C, 72.38; H, 4.37\%; Found: C, 72.33; H, 4.46\%.

4-(2-Methoxybenzyl)-3-(3,5-dimethyl-1 H-pyrazol-1-yl)-6phenylpyridazine (8a): Prepared from acetyl acetone brown, crystals in $80 \%$ yield, $\mathrm{mp} 83-4^{\circ} \mathrm{C}$ (benzene); Mass spectrum $m / z 371\left(\mathrm{M}^{+}+1,28.35\right), 370\left(\mathrm{M}^{+}, 100\right), \mathrm{IR}: 1596(\mathrm{C}=\mathrm{N})$ and $2926 \mathrm{~cm}^{-1}$ (CH aliphatic), ${ }^{1} \mathrm{HNMR}\left(\mathrm{CDCl}_{3}, 300 \mathrm{MHz}\right) \delta 2.22-$ $2.36\left(\mathrm{~s}, 6 \mathrm{H}, 2 \mathrm{CH}_{3}\right), 3.82\left(\mathrm{~s}, 3 \mathrm{H}, \mathrm{OCH}_{3}\right), 4.14\left(\mathrm{~s}, 2 \mathrm{H}, \mathrm{CH}_{2}\right), 6.04-$ $6.84(\mathrm{~s}, 2 \mathrm{H}, 2 \mathrm{CH}$ hetero) and 7.26-8.02 (m, 9H, 2Ar-H). Anal. Calcd for $\mathrm{C}_{23} \mathrm{H}_{22} \mathrm{~N}_{4} \mathrm{O}$ : C, $74.57 ; \mathrm{H}, 5.99 ; \mathrm{N}, 15.12 \%$. Found: $\mathrm{C}$, $74.30 ; \mathrm{H}, 5.80 ; \mathrm{N}, 15.22 \%$.

4-(2-Methoxybenzyl)-3-(3,5-dimethyl-1 H-pyrazol-1-yl)-6methylphenylpyridazine (8b): Prepared from acetyl acetone, brown crystals in $52 \%$ yield, mp $91-2{ }^{\circ} \mathrm{C}$ (benzene); Mass spectrum $m / z 386\left(\mathrm{M}^{+}+2,2.83\right), 385\left(\mathrm{M}^{+}+1,4.03\right), 384\left(\mathrm{M}^{+}, 12.68\right)$, IR: $1590(\mathrm{C}=\mathrm{N})$ and $2919 \mathrm{~cm}^{-1}$ (CH aliphatic). Anal. Calcd for $\mathrm{C}_{24} \mathrm{H}_{24} \mathrm{~N}_{4} \mathrm{O}: \mathrm{C}, 74.97 ; \mathrm{H}, 6.29 ; \mathrm{N}, 14.57 \%$. Found: C, 74.90; H, $6.00 ; \mathrm{N}, 14.38 \%$.

4-(2-Methoxybenzyl)-3-(3,5-dimethyl-1H-pyrazol-1-yl)-6chlorophenylpyridazine (8c): Prepared from acetyl acetone, brown crystals in $44 \%$ yield, $\mathrm{mp} 132{ }^{\circ} \mathrm{C}$ (benzene); Mass spectrum $m / z 407\left(\mathrm{M}^{+}+3,11.50\right), 406\left(\mathrm{M}^{+}+2,36.70\right), 405\left(\mathrm{M}^{+}+1\right.$, 29.85), $404\left(\mathrm{M}^{+}, 100\right)$, IR: $1595(\mathrm{C}=\mathrm{N})$ and $2922 \mathrm{~cm}^{-1}(\mathrm{CH}$ aliphatic). Anal. Calcd for $\mathrm{C}_{23} \mathrm{H}_{21} \mathrm{~N}_{4} \mathrm{OCl}$ : C, 68.23; H, 5.23; N, 13.84; Cl, 8.76\%. Found: C, 67.89; H, 5.54; N, 13.80; Cl, 8.77\%.

4-(2-Methoxybenzyl)-3-(3,5-dimethyl-1 H-pyrazol-1-yl)-6methoxyphenylpyridazine (8d): Prepared from acetyl acetone brown crystals in $70 \%$ yield, mp $75-6{ }^{\circ} \mathrm{C}$ (benzene); Mass spectrum $m / z 402\left(\mathrm{M}^{+}+2,8.24\right), 401\left(\mathrm{M}^{+}+1,15.29\right), 400\left(\mathrm{M}^{+}\right.$, 24.71), IR: $1605(\mathrm{C}=\mathrm{N})$ and $2931 \mathrm{~cm}^{-1}$ (CH aliphatic); ${ }^{1} \mathrm{HNMR}$ $\left(\mathrm{CDCl}_{3}, 300 \mathrm{MHz}\right) \delta 2.18-2.34\left(\mathrm{~s}, 6 \mathrm{H}, 2 \mathrm{CH}_{3}\right), 3.69-3.85(\mathrm{~s}$, $\left.6 \mathrm{H}, 2 \mathrm{OCH}_{3}\right), 4.09\left(\mathrm{~s}, 2 \mathrm{H}, \mathrm{CH}_{2}\right), 6.01-6.79(\mathrm{~s}, 2 \mathrm{H}, 2 \mathrm{CH}$ hetero) and 6.81-7.98 (m, 8H, 2Ar-H). Anal.Calcd for $\mathrm{C}_{24} \mathrm{H}_{24} \mathrm{~N}_{4} \mathrm{O}_{2}$ : C, 71.98; H, 6.04; N, 13.99\%. Found: C, 71.80; H, 5.70; N, $14.32 \%$.

General procedure for the preparation of tetrazolo from 1[4-(2-Methoxy benzyl)-6-aryl pyridazin-3(2H)-ylidene] hydrazines 1a-d and nitrous acid. To a solution of 1a-d (1 mmole) in $1.25 \mathrm{~N} \mathrm{HCl}(20 \mathrm{~mL})$, was added sod. nitrite solution (1 mmole) at $(0-5 \mathrm{C})$ during 30 minutes with stirring. The reaction mixture was kept for $2 \mathrm{hrs}$ at room temperature, diluted with water and filtered. The solid product obtained was dried and crystallized from the appropriate solvent.

8-(2-Methoxybenzyl)-6-phenyl tetrazolo pyridazine (9a): Pale brown crystals in $60 \%$ yield, mp $121-2{ }^{\circ} \mathrm{C}$ (benzene); Mass spectrum $m / z 318\left(\mathrm{M}^{+}+1,13.38\right), 317\left(\mathrm{M}^{+}\right.$, 57.66), IR: 1164 (tertazole), $1568(\mathrm{~N}=\mathrm{N}), 1597(\mathrm{C}=\mathrm{N})$ and $2077 \mathrm{~cm}^{-1}\left(\mathrm{~N}_{3}\right.$ azide). ${ }^{1} \mathrm{HNMR}\left(\mathrm{CDCl}_{3}, 300 \mathrm{MHz}\right) \delta 3.82\left(\mathrm{~s}, 3 \mathrm{H}, \mathrm{OCH}_{3}\right), 4.55$ $\left(\mathrm{s}, 2 \mathrm{H}, \mathrm{CH}_{2}\right), 6.94(\mathrm{~s}, 1 \mathrm{H}, \mathrm{CH}$ hetero) and 6.96-7.97 (m, 9H, 2Ar-H). Anal. Calcd for $\mathrm{C}_{18} \mathrm{H}_{15} \mathrm{~N}_{5} \mathrm{O}: \mathrm{C}, 68.13 ; \mathrm{H}, 4.76$; N, $22.07 \%$. Found: C, 67.92; H, 4.56; N, 22.10\%.
8-(2-Methoxybenzyl)-6-methylphenyl tetrazolo pyridazine (9b): Pale brown crystals in $85 \%$ yield, mp $124-5^{\circ} \mathrm{C}$ (benzene); Mass spectrum $m / z 332\left(\mathrm{M}^{+}+1,1.93\right), 331\left(\mathrm{M}^{+}, 5.71\right)$, IR: 1189 (tertazole), $1574(\mathrm{~N}=\mathrm{N}) 1601(\mathrm{C}=\mathrm{N})$ and $2047 \mathrm{~cm}^{-1}\left(\mathrm{~N}_{3}\right.$ azide), Anal. Calcd. for $\mathrm{C}_{19} \mathrm{H}_{17} \mathrm{~N}_{5} \mathrm{O}$ : C, 68.87; H, 5.17; N, 21.13\%. Found: C, 68.60; H, 5.43; N, 21.25\%.

8-(2-Methoxy benzyl)-6-chlorophenyl tetrazolo pyridazine (9c): Pale brown crystals in $45 \%$ yield, mp $141-2^{\circ} \mathrm{C}$ (benzene); Mass spectrum $m / z 354\left(\mathrm{M}^{+}+3,8.19\right), 353\left(\mathrm{M}^{+}+2,27.27\right), 352$ $\left(\mathrm{M}^{+}+1,18.47\right), \mathrm{IR}: 1178$ (tertazole), $1567(\mathrm{~N}=\mathrm{N}), 1595(\mathrm{C}=\mathrm{N})$ and $2034 \mathrm{~cm}^{-1}\left(\mathrm{~N}_{3}\right.$ azide). Anal. Calcd for $\mathrm{C}_{18} \mathrm{H}_{14} \mathrm{~N}_{5} \mathrm{OCl}$ : $\mathrm{C}$, 61.46; H, 4.01; N, 19.91; Cl, 10.08\%. Found: C, 61.54; H, 3.70; N, 19.90; Cl, 10.12\%.

8-(2-Methoxybenzyl)-6-methoxyphenyl tetrazolopyridazine (9d): Pale brown crystals in $40 \%$ yield, mp $195-6^{\circ} \mathrm{C}$ (benzene); Mass spectrum $m / z 348\left(\mathrm{M}^{+}+1,3.92\right), 347\left(\mathrm{M}^{+}, 6.25\right)$, IR: 1186 (tertazole), $1573(\mathrm{~N}=\mathrm{N}), 1602(\mathrm{C}=\mathrm{N})$ and $2036 \mathrm{~cm}^{-1}\left(\mathrm{~N}_{3}\right.$ azide). ${ }^{1} \mathrm{HNMR}\left(\mathrm{CDCl}_{3}, 300 \mathrm{MHz}\right) \delta 3.80-3.91\left(\mathrm{~s}, 6 \mathrm{H}, 2 \mathrm{OCH}_{3}\right)$, $4.53\left(\mathrm{~s}, 2 \mathrm{H}, \mathrm{CH}_{2}\right), 6.94(\mathrm{~s}, 1 \mathrm{H}, \mathrm{CH}$ hetero) and 6.96-7.94 (m, $8 \mathrm{H}, 2 \mathrm{Ar}-\mathrm{H})$. Anal. Calcd for $\mathrm{C}_{19} \mathrm{H}_{17} \mathrm{~N}_{5} \mathrm{O}_{2}: \mathrm{C}, 65.69 ; \mathrm{H}, 4.93 ; \mathrm{N}$, $20.16 \%$. Found: C, 65.80; H, 4.90; N, 20.12\%.

General procedure for the preparation of acrylohydrazines 10a-c from 1-[4-(2-methoxy benzyl)-6-arylpyridazin-3(2H)ylidene] hydrazines 1a-c and acryloyl chloride. A solution of acryloyl chloride (1 mmole) in ( $20 \mathrm{~mL})$ methylene chloride was added drop wise to a solution of 1 a-c $(1 \mathrm{~mole})$ in $(30 \mathrm{~mL}) \mathrm{me}-$ thylene chloride. The reaction mixture was stirred for $1 \mathrm{hr}$, then extracted with $(5 \mathrm{~mL}) \mathrm{HCl},(5 \mathrm{~mL})$ of $5 \% \mathrm{NaOH}$ and finally with $(5 \mathrm{~mL})$ of water. The methylene dichloride layer was dried over anhydrous $\mathrm{Na}_{2} \mathrm{SO}_{4}$. The solid product obtained after evaporation under reduced pressure was crystallized from the proper solvent.

1-[4-(2-Methoxybenzyl)-6-phenylpyridazin-3-(2H)-ylidene] acrylohydrazine (10a): Pale brown crystals in $80 \%$ yield, mp 154 - $5{ }^{\circ} \mathrm{C}$ (ethanol); Mass spectrum $m / z 361\left(\mathrm{M}^{+}+1,3.38\right), 360$ $\left(\mathrm{M}^{+}, 11.21\right)$, IR: $1537(\mathrm{C}=\mathrm{C}), 1603(\mathrm{C}=\mathrm{N}), 1661(\mathrm{C}=\mathrm{O}), 2929$ (CH aliphatic) and $3161 \& 3314 \mathrm{~cm}^{-1}(2 \mathrm{NH}) .{ }^{1} \mathrm{HNMR}\left(\mathrm{CDCl}_{3}\right.$, $300 \mathrm{MHz}) \delta 3.88\left(\mathrm{~s}, 3 \mathrm{H}, \mathrm{OCH}_{3}\right), 3.95\left(\mathrm{~s}, 2 \mathrm{H}, \mathrm{CH}_{2}\right)$ 5.65-5.68 $\left(\mathrm{d}, 2 \mathrm{H}, \mathrm{CH}_{2}\right), 6.38-6.39(\mathrm{~d}, 1 \mathrm{H}, \mathrm{CH}), 6.92(\mathrm{~s}, 1 \mathrm{H}, \mathrm{CH}$ hetero), 6.95-7.44 (m, 9H, 2Ar-H) and 7.85-7.88 (s, 2H, 2NH). Anal. Calcd for $\mathrm{C}_{21} \mathrm{H}_{20} \mathrm{~N}_{4} \mathrm{O}_{2}$ : C, 69.98; H, 5.59; N, 15.55\%. Found: C, 69.64; H, 5.42; N, 15.25\%.

1-[4-(2-Methoxybenzyl)-6-methylphenylpyridazin-3-(2H)ylidene] acrylohydrazine (10b): Pale brown crystals in $36 \%$ yield, $\mathrm{mp} 117-8^{\circ} \mathrm{C}$ (ethanol); Mass spectrum $m / z 375\left(\mathrm{M}^{+}+1\right.$, 0.49), $374\left(\mathrm{M}^{+}, 1.48\right)$, IR: $1559(\mathrm{C}=\mathrm{C}), 1601(\mathrm{C}=\mathrm{N}), 1672(\mathrm{C}=\mathrm{O})$, 2925 (CH aliphatic) and broad band at $3221 \mathrm{~cm}^{-1}$ (2NH). Anal. Calcd for $\mathrm{C}_{22} \mathrm{H}_{22} \mathrm{~N}_{4} \mathrm{O}_{2}$ : C, 70.57; H, 5.92\%. Found: C, 70.35; $\mathrm{H}, 6.35 \%$.

1-[4-(2-Methoxybenzyl)-6-chlorophenylpyridazin-3-(2H)ylidene] acrylohydrazine (10c): Pale brown crystals in $40 \%$ yield, mp $109-110^{\circ} \mathrm{C}$ (ethanol); Mass spectrum $m / z 395\left(\mathrm{M}^{+}+1\right.$, 0.52), $394\left(\mathrm{M}^{+}, 1.46\right), \mathrm{IR}: 1556(\mathrm{C}=\mathrm{C}), 1595(\mathrm{C}=\mathrm{N}), 1662(\mathrm{C}=\mathrm{O})$, 2926 (CH aliphatic) and broad band at $3171 \mathrm{~cm}^{-1}(2 \mathrm{NH})$. Anal. Calcd for $\mathrm{C}_{21} \mathrm{H}_{19} \mathrm{~N}_{4} \mathrm{O}_{2} \mathrm{Cl}$ : C, 63.88; H, 4.85; N, 14.19; $\mathrm{Cl}, 8.98 \%$. Found: C, 63.66; H, 5.04; N, 14.23; Cl, 8.57\%.

General procedure for the preparation of hydrazides 11a-c and 12a,b from 1-[4-(2-methoxy benzyl)-6-arylpyridazin-3(2H)- 
ylidene] hydrazines 1a-c and acid chlorides. A mixture of 1a-c (1 mmole) with the appropriate chloride ( 1 mmole) was refluxed in $(10 \mathrm{~mL})$ dry benzene in presence of few drops of pyridine for $8 \mathrm{hrs}$. After cooling to room temperature, the solid product obtained was crystallized form the proper solvent.

$\mathrm{N}$-[4-(2-Methoxybenzyl)-6-phenylpyridazin-3(2H)-ylidene] furan-2-carbohydrazide (11a): Prepared from 2-furoyl chloride, dark brown crystals in $80 \%$ yield, mp $157-8^{\circ} \mathrm{C}$ (benzene); Mass spectrum $m / z 427\left(\mathrm{M}^{+}+27,1.25\right), 351(100), 305$ (3.16), 248 (12.84), 228156 (3.04), 115 (9.46), 77 (94.72), IR:1598 (C=N), $1705(\mathrm{C}=\mathrm{O}), 3116(\mathrm{NH})$ and $3403 \mathrm{~cm}^{-1}(\mathrm{OH}) .{ }^{1} \mathrm{HNMR}\left(\mathrm{CDCl}_{3}\right.$, $300 \mathrm{MHz}) \delta 3.82\left(\mathrm{~s}, 3 \mathrm{H}, \mathrm{OCH}_{3}\right), 4.56\left(\mathrm{~s}, 2 \mathrm{H}, \mathrm{CH}_{2}\right), 6.66(\mathrm{~s}, 1 \mathrm{H}$, $\mathrm{CH}$ hetero), 6.93-6.96 (d, 1H, CH hetero), 6.98-7.10 (t, $1 \mathrm{H}, \mathrm{CH}$ hetero), 7.23-7.13 (d, 1H, CH hetero), 7.29-7.89 (m, 9H, 2Ar-H), $8.48(\mathrm{~s}, 2 \mathrm{H}, 2 \mathrm{NH})$ and $8.82(\mathrm{~s}, 1 \mathrm{H}, \mathrm{OH}$ of amide). Anal. Calcd for $\mathrm{C}_{23} \mathrm{H}_{20} \mathrm{~N}_{4} \mathrm{O}_{3}$ : C, 68.99; H, 5.03; N, 13.99\%. Found: C, 68.72; H, 4.95 ; N, $13.66 \%$.

$\mathrm{N}^{\mathrm{N}}$-[4-(2-Methoxy benzyl)-6-methylphenylpyridazin-3(2H)ylidene] furan-2-carbohydrazide (11b): Prepared from 2-furoyl chloride, pink crystals in $78 \%$ yield, mp $201-2{ }^{\circ} \mathrm{C}$ (benzene); Mass spectrum $m / z 397\left(\mathrm{M}^{+}-17,1.56\right), 365$ (100), 350 (0.67), 290 (6.23), 248 (16.30), 193 (2.40), 140 (8.08), 115 (17.35), 91 (99.54), 65 (71.07), IR: $1598(\mathrm{C}=\mathrm{N}), 1657(\mathrm{C}=\mathrm{O}), 3120(\mathrm{NH})$ and $3423 \mathrm{~cm}^{-1}(\mathrm{OH})$. Anal. Calcd for $\mathrm{C}_{24} \mathrm{H}_{22} \mathrm{~N}_{4} \mathrm{O}_{3}: \mathrm{N}, 13.52 \%$. Found: N, 13.77\%.

$N$-[4-(2-Methoxybenzyl)-6-chlorophenylpyridazin-3(2H)ylidene] furan-2-carbohydrazide (11c): Prepared from 2-furoyl chloride, pink crystals in $37 \%$ yield, mp $189-190{ }^{\circ} \mathrm{C}$ (benzene); Mass spectrum $m / z 432\left(\mathrm{M}^{+}-2,0.13\right)$, IR: $1597(\mathrm{C}=\mathrm{N})$, $1651(\mathrm{C}=\mathrm{O}), 3101(\mathrm{NH}), 3423 \mathrm{~cm}^{-1}(\mathrm{OH})$, and Anal. Calcd for $\mathrm{C}_{23} \mathrm{H}_{19} \mathrm{~N}_{4} \mathrm{O}_{3} \mathrm{Cl}: \mathrm{N}, 12.88 ; \mathrm{Cl}, 8.15 \%$. Found: N, 13.01; Cl, 8.21\%.

$\boldsymbol{N}$-[4-(2-Methoxybenzyl)-6-phenylpyridazin-3(2H)-ylidene]-2-chloroacetohydrazide (12a): Prepared from chloroacetyl chloride, Pale brown crystals in $75 \%$ yield, mp $161-2{ }^{\circ} \mathrm{C}$ (benzene); Mass spectrum $m / z 382\left(\mathrm{M}^{+}, 0.41\right)$. IR: $1600(\mathrm{C}=\mathrm{N})$, $1654(\mathrm{C}=\mathrm{O})$ and $3422 \mathrm{~cm}^{-1}(\mathrm{NH}) .{ }^{1} \mathrm{HNMR}\left(\mathrm{CDCl}_{3}, 300 \mathrm{MHz}\right)$ $\delta 3.79\left(\mathrm{~s}, 3 \mathrm{H}, \mathrm{OCH}_{3}\right), 4.48\left(\mathrm{~s}, 2 \mathrm{H}, \mathrm{CH}_{2}\right), 5.16\left(\mathrm{~s}, 2 \mathrm{H}, \mathrm{CH}_{2}\right), 6.89$ (s, 1H, CH hetero), 6.92-7.47 (m, 9H, 2Ar-H) and 7.82-7.85 (s, $2 \mathrm{H}, 2 \mathrm{NH})$. Anal. Calcd for $\mathrm{C}_{20} \mathrm{H}_{19} \mathrm{~N}_{4} \mathrm{O}_{2} \mathrm{Cl}$ : N, 14.63; $\mathrm{Cl}, 9.26 \%$. Found: N, 14.36; Cl, 9.26\%.

$N$ '-[4-(2-Methoxybenzyl)-6-phenylpyridazin-3(2H)-ylidene]-2-chloroacetohydrazide (12b): Prepared from chloroacetyl chloride, pale yellow crystals in $50 \%$ yield , mp $134{ }^{\circ} \mathrm{C}$ (benzene); Mass spectrum $m / z 397\left(\mathrm{M}^{+}+1,11.22\right)$, IR: 1602 $(\mathrm{C}=\mathrm{N}), 1654(\mathrm{C}=\mathrm{O})$ and $3450 \mathrm{~cm}^{-1}(\mathrm{NH})$. Anal. Calcd for $\mathrm{C}_{21} \mathrm{H}_{21} \mathrm{~N}_{4} \mathrm{O}_{2} \mathrm{Cl}: \mathrm{N}, 14.12 ; \mathrm{Cl}, 8.93 \%$. Found: N, 14.20; $\mathrm{Cl}, 8.95 \%$.

Enone reaction of $5 \mathrm{a}$ and $10 \mathrm{a}$ with maleic anhydride. Fusion of compound $\mathbf{5 a}$ and/or 10a (1 mmole) with maleic anhydride ( $1 \mathrm{mmole}$ ) for $3 \mathrm{hrs}$ above its melting point. The solid obtained on cooling was crystallized from the appropriate solvent.

1-[4-(2-Methoxybenzyl)-6-phenylpyridazin-3(2H)-ylidene]2-(7-methyl-2,4-dioxo-3-oxa-bicycle $[3,2,0]$ heptan-6-carbaldehyde) hydrazine (6): Dark brown crystals in 65\% yield, mp $103-4{ }^{\circ} \mathrm{C}$ (benzene). IR: $1601(\mathrm{C}=\mathrm{N}), 1650 \& 1729(2 \mathrm{C}=\mathrm{O})$ and $3421 \mathrm{~cm}^{-1}(\mathrm{NH})$. Anal. Calcd for $\mathrm{C}_{26} \mathrm{H}_{24} \mathrm{~N}_{4} \mathrm{O}_{4}$ : C, 68.41; H, 5.30; N, 12.27\% .Found: C, 68.62; H, 5.58; N, 12.01\%.

$\mathrm{N}$-[4-(2-Methoxybenzyl)-6-phenylpyridazin3(2H)-ylidene]2,4-dioxo-3-oxa-bicyclo [3,2,0] heptan-6-carbohydrazide (13):
Dark brown crystals in $35 \%$ yield, mp $181-2{ }^{\circ} \mathrm{C}$ (ethanol); Mass spectrum $m / z 457\left(\mathrm{M}^{+}-1,6.73\right)$, IR: $1600(\mathrm{C}=\mathrm{N}), 1655 \&$ $1727(2 \mathrm{C}=\mathrm{O})$ and $3400 \mathrm{~cm}^{-1}(2 \mathrm{NH}) .{ }^{1} \mathrm{HNMR}\left(\mathrm{CDCl}_{3}, 300 \mathrm{MHz}\right)$

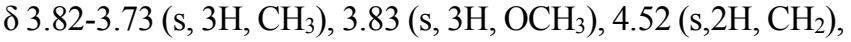
5.87-5.85 (t, 2H, $\left.\mathrm{CH}_{2}\right), 6.91$ (s, 1H, CH hetero), 6.93-6.96 (q, 1H, $\mathrm{CH}), 6.97-7.12$ (q, 1H, CH hetero), 7.20-7.23 (t, 1H, CH hetero), 7.26-7.51 (m, 9H, 2Ar-H) and 7.87-7.84 (s, 2H, 2NH). Anal. Calcd for $\mathrm{C}_{25} \mathrm{H}_{22} \mathrm{~N}_{4} \mathrm{O}_{5}$ : C, 65.49; $\mathrm{H}, 4.84 ; \mathrm{N}, 12.22$. Found: $\mathrm{C}$, 65.60; H, 5.10; N, 12.13 .

Synthesis of silver-nanoparticles (Ag-NPs). The silver colloid was prepared by using chemical reduction method reported. ${ }^{36}$ Silver nitrate $(>99 \%)$ and trisodium citrate dihydrate $(99.0 \%)$ were purchased from Aldrich. All descriptions of water below refer to Nanopure deionized water (electrical resistance $>18.4$ $\mathrm{M} \Omega-\mathrm{cm}$ ), produced. Silver nanoparticles were prepared by citrate reduction of silver nitrate. $\mathrm{AgNO}_{3}(17.0 \mathrm{mg})$ was dissolved in $100 \mathrm{~mL}$ water in a $250 \mathrm{~mL}$ tri-neck flask. The solution was heated to boiling with a hemisphere heating mantle under vigorous magnetic stirring. After boiling for 2 minutes, an aqueous solution of sodium citrate $(35 \mathrm{mM}, 10 \mathrm{~mL})$ was rapidly added to the flask. The solution gradually turned yellow within a few minutes, indicating the formation of Ag nanoparticles. The solution was kept boiling for an additional 6 minutes. After that, the heating mantle was removed, and the solution was allowed to cool. After cooling, a solution of compounds $4 \mathbf{a}, \mathbf{8 a}, \mathbf{b}, \mathbf{1 0 a}$, $\mathbf{1 1 b}$ and $\mathbf{1 2 b}\left(3 \times 10^{-3} \mathrm{gm}\right.$ soluble in $5 \mathrm{~mL}$ ethanol $)$ was stirred with Ag-NPs solution ( $10 \mathrm{~mL}$ ) for $24 \mathrm{hrs}$, after that the reaction mixture was evaporated and the solid product was obtained in the nano form.

\section{References and Note}

1. Kelly, K. L.; Coronado, E.; Zhao, L. L.; Schatz, G. C. J. Phys. Chem. 2003, B 107(3), 668.

2. Nurmi, J. T.; Tratnyek, P. G.; Sarathy, V.; Baer, D. R.; Amonette, J. E.; Pecher, K.; Wang, C.; Linehan, J. C.; Matson, D. W.; Penn, R. L.; Driessen, M. D. Environ. Sci. Technol. 2004, 39(5), 1221.

3. Sondi, I.; Salopek-Sondi, B. J. Colloid Interface Sci. 2004, 275(1), 177.

4. Navarro, E.; Piccapietra, F.; Wagner, B.; Marconi, F.; Kaegi, R.; Odzak, N.; Sigg, L.; Behra, R. Environ. Sci. Technol. 2008, 42(23), 8959.

5. Carlson, C.; Hussain, S. M.; Schrand, A. M.; Braydich-Stolle, K. L.; Hess, K. L.; Jones, R. L.; Schlager, J. J. J. Phys. Chem. B 2008, $112(43), 13608$.

6. Choi, O.; Hu, Z. Sci. Technol. 2008, 42, 4583.

7. Jiang, W.; KimBetty, Y. S.; Rutka, J. T.; ChanWarren, C. W. Nat. Nanotechnol. 2008, 3(3), 145.

8. Choi, O.; Deng, K.; Kim, N.; Ross, L.; Hu, Z. Water Res. 2008, 42, 3066.

9. Kim, T. N.; Feng, Q. L.; Kim, J. O.; Wu, J.; Wang, H.; Chen, G. C.; Cui, F. Z. J. Mater. Sci. Mater. Med. 1998, 9, 129.

10. Uchida, M. Chem. Ind. 1995, 46, 48.

11. Grier, N. Philadelphia 1983, 375.

12. Lehninger, A.; Nelson, D.; Cox, M. Principles of Biochemistry, 2nd ed.; Worth: New York, 1993.

13. Liau, S.; Read, D.; Pugh, W.; Furr, J.; Russell, A. Lett. Appl. Microbiol. 1997, 25, 279.

14. Davies, R.; Etris, S. Catal. Today 1997, 35, 87.

15. Kowshik, M.; Ashtaputre, S.; Kharrazi, S. Nanotechnology 2003, 14,95 .

16. Souza, G. I. H.; Marcato, P. D.; Durán, N.; Esposito, E. In IXNatio- 
nal Meeting of Environmental Microbiology; Curtiba: PR (Brazil), 2004 Abstr.; 25.

17. Duran, N.; Marcato, P. D.; Alves, O. L.; Souza, G. J. Nanotechnology 2005, 3, 8 .

18. Cho, K. H.; Park, J. E.; Osaka,T.; Park, S. G. Electrochimica Acta 2005, 51, 956.

19. Wright, G. D. Chem. Biol. 2000, 7, 127.

20. Wright, G. D. Adv. Drug Deliv. Rev. 2005, 57, 1451.

21. Zou, X. J.; Lai, L. H.; Jin, G. Y.; Zhang, Z. X. J. Agric. Food Chem. 2002, 50(6), 3757.

22. Mark, S.; John, C. V.; Todd, A. B.; Jennifer, M.; Mattew, J. L.; Adam, G.; Biswanath, D.; Surian, E. V.; Lily, C. H.; Jeff, R.; Steve, B.; Eric, S.; Michael, J. Bioorg. Med. Chem. Lett. 2006, 16, 4257.

23. Kleemann, A. J. Pharmazeutische Wirkstoffe, Syntheses Patente, Anweedungen; Thieme Verlag: Stuttgart, 1978.

24. Elias, O.; Karolyhazy, L.; Horvath, G.; Harmt, V.; Matyus, P. J. Mol. Struct. Theochem. 2003, 666-667, 625.

25. Orru, R. V. A.; De Greef, M. Synthesis 2003, 10, 1471.

26. Bienaymé, H.; Oddon, C. G.; Schmitt, P. Chem. Eur. J. 2000, 6, 332.

27. (a) Vigorita, M. G.; Ottana, R.; Monforte, F.; Maccari, R.; Trovato, A.; Monforte, M. T.; Taviano, M. F. Bioorg. Med. Chem. Lett. 2001, 11, 2791. (b) Chande, M. S.; Suryanarayan,V.J. Chem. Res. 2005, 6, 345. (c) Kavitha, C. V.; Basappa, S.; Swamy, N.; Mantelingu, K.; Doreswamy, S.; Sridhar, M. A.; Prasad, S.; Rangappa, K. S. Bioorg. Med. Chem. 2006, 14, 2290. (d) Shiradkar, M.; Shivaprasad, H. N. Asian J. Chem. 2006, 18, 331.

28. Mohamed, M. I. J. Bulg. Chem. Commun. 2004, 36(4), 241.
29. Mohamed, M. I.; Zaky, H.T.; Kandile, N. G. J. Chin Chem. Soc. 2004, 51, 963.

30. Mohamed, M. I. J. Bulg. Chem .Commun. 2007, 39(2), 152.

31. Kandile, N. G.; Mohamed, M. I.; Zaky, H. T.; Mohamed, H. M. European Journal of Medicinal Chemistry 2009, 44, 1989.

32. Mohamed, M. I.; Zaky, H. T.; Mohamed, H. M.; Kandile, N. G. Afinidad 2005, 62(515), 48.

33. Haider, N.; Kabicher, T.; Kaferbock, J.; Plank, A. Molecules 2007, 12,1900

34. Cmoch, P. Magn. Reson. Chem. 2002, 40, 507.

35. Katrusiak, A.; Skierska, U.; Katrusiak, A. J. Mol. Struc. 2005, 751, 71.

36. Yakutik, I. M.; Shevchenko, G. P.; Rakhmanov, S. K. The Formation of Mono Disperse Spherical Silver Particles, Colloids and Surfaces A:Physicochemical and Engineering Aspects; 2004; pp 175.

37. Henglein, A. J. Phys. Chem. 1993, 97, 5457.

38. Sastry, M.; Mayya, K. S.; Bandyopadhyay, K. Colloid. Surf. A 1997, 127, 221.

39. Sastry, M.; Patil, V.; Sainkar, S. R. J.Phys. Chem. B 1998, 102, 1404.

40. Jawetz, E.; Melnick, J. L.; Adelberg, E. A. Review of Medical Microbiology; Lang Medical Publication: Los Altos, California, 1974.

41. Grayer, J. R.; Harbone, J. B. Phytochemistry 1994, 37, 19.

42. Muanza, D. N.; Kim, B. W.; Euler, K. L.; Williams, L. Pharm. Biol. 1994, 32, 337.

43. Irobi, O. N.; Moo-Young, M.; Anderson, W. A. Pharm. Biol. 1996, $34,87$. 